\title{
A WEIGHTED RESIDUAL PARABOLIC ACCELERATION TIME INTEGRATION METHOD FOR PROBLEMS IN STRUCTURAL DYNAMICS
}

\author{
S. H. RAZAVI ${ }^{1}$, A. ABOLMAALI ${ }^{1}$, AND M. GHASSEMIEH ${ }^{2}$
}

\begin{abstract}
In the proposed method, the variation of displacement in each time step is assumed to be a fourth order polynomial in time and its five unknown coefficients are calculated based on: two initial conditions from the previous time step; satisfying the equation of motion at both ends of the time step; and the zero weighted residual within the time step. This method is non-dissipative and its dispersion is considerably less than in other popular methods. The stability of the method shows that the critical time step is more than twice of that for the linear acceleration method and its convergence is of fourth order.
\end{abstract}

2000 Mathematics Subject Classification: 65L05, 65D30, 37M10.

Keywords: structural dynamics, time integration, numerical stability, numerical accuracy, convergence, dissipation, dispersion, implicit method, explicit method.

\section{Introduction}

Time integration methods are the most suitable methods for nonlinear problems in structural dynamics and for dynamic analysis of very large structures. Among different methods, those related to the Newmark method are the most common ones. The time integration algorithms are categorized based on the satisfying of the equation of motion either at the beginning or at the end of each time step. The algorithms in which the equation of motion is satisfied at the beginning of the time step, to calculate the values of the displacement and velocity at the end of the time step, are called explicit methods. On the other hand, algorithms in which the equation of motion is satisfied at the end of each time step are called implicit methods. The efficiency of each method depends on the convergence, consistency, and stability of the method. A review of several implicit and explicit methods is found in [7-12].

The explicit-implicit analysis technique is often used when the domain of the system under consideration contains both stiff and flexible components, for example, in fluid-structure interaction problems. For fluid and structure regions, explicit and implicit methods are used, respectively. In this method, in each time step, the explicit regions are first analyzed, and the results are used as the boundary conditions for the implicit regions, which share a boundary with the explicit regions as shown in $[13,14]$.

For accuracy evaluation of the time integration methods, usually the following quantities are determined: numerical damping (dissipation), periodic error (dispersion), and overshoot.

\footnotetext{
${ }^{1}$ Department of Civil and Environmental Engineering, University of Texas at Arlington, Arlington, TX 76019. E-mail: razavihadi@yahoo.com

${ }^{2}$ Department of Civil Engineering, Faculty of Engineering, University of Tehran, Tehran, Iran.
} 
Some methods have a numerical damping that causes a response of the structure to the damping. For instance, in the absence of physical damping, the free vibration response shows a damped behavior, which is unexpected. This numerical damping is a function of the time step of integration.

Dispersion introduces numerical errors by elongating or shortening the natural period of vibration compared to its exact value. The numerical error due to overshoot causes an amplitude reduction in the first few time steps, which was first observed in the Wilson- $\theta$ method and later was investigated for other methods in [17]. The presence of this error is due to the fact that short-term solutions are a function of the norm of the amplification matrix, while long-term solutions depend on the spectral radius. Therefore, for a stable integration method, the norm of the amplification matrix can be a large number, even though the spectral radius is small.

Stability of a method requires bounded solutions (limited errors). Indeed, the stability of a method depends on the degree of error propagation from one time step to the next one, and if a considerable amount of error propagates to the next time step from the previous ones, after a while, the solution will diverge from the exact solution. In conditionally stable methods, this instability happens when the size of the time step is more than a specific value called the critical time step, while for unconditionally stable algorithms, instability never occurs regardless of the time step size. A systematic procedure was proposed in [18] to investigate the stability and accuracy of time integration methods. This procedure is used to evaluate the proposed method.

According to the Lax theorem, there is a relationship between the following numerical notions: convergence, consistency and stability. It says consistency and stability guarantee convergence of an algorithm [19]. Almost all of the explicit methods are conditionally stable and for a few of them with unconditional stability, the consistency is conditional.

The explicit methods require less calculation within each time step with a large number of time steps, while implicit methods require more calculation in each time step with a smaller number of time steps. Generally, it has been shown that the implicit methods are more accurate than the explicit ones $[7,11]$.

\section{Proposed method}

In the proposed method, the displacement in each time step is assumed to be a fourth order complete polynomial which means quadratic variation of acceleration with time. This displacement function contains five constants that are calculated based on the following conditions: 1) two initial conditions from the previous time step are used; 2) the equation of motion is exactly satisfied at both ends of the time step, and 3) the approximate solution in each time step is forced to have a zero average error in satisfying the equation of motion (a weighted residual equation with the weight function of unity). When the average residue in each time step is forced to vanish, it makes the approximate solution take one of the two forms: either have a zero residue everywhere in the time step, or have both negative and positive errors which are small for smooth functions within a short time step The aforementioned five conditions provide an adequate number of equations to be solved for the coefficients of the assumed polynomial for the displacement function from which the values of the displacement and velocity at the end of the time step can be calculated.

Also, the amplification matrix is derived and the spectral radius (norm of the complex eigenvalue of the amplification matrix) is calculated to evaluate the stability of the proposed 
method, which shows that the algorithm is stable for time steps (CTS) of up to $1.24 T$ ( $T=$ natural period of vibration). This proposed CTS is compared to the Newmark linear acceleration method with CTS $=0.55 T$, the central finite difference method with CTS $=$ $0.32 T$, and the Fox - Goodwin method with CTS $=0.39 T$. This indicates that this method possesses a stability range, which is superior to other conditionally stable methods with the CTS $=1.24 T$, which is uniquely greater than the period of vibration, $T$, of the system. To compare the accuracy of this method with other methods in the literature, dissipation and dispersion errors are obtained which indicates a high degree of accuracy. This method is nondissipative and its dispersion error is significantly lower than in the other methods. In addition, as an example, the spectral displacement for the harmonic load with this method is compared with several well-known methods to confirm a higher degree of accuracy of the proposed method. To evaluate the order of convergence of the method, harmonic loads are applied and for a fixed time, with a different number of time steps, the displacement errors at that time are calculated, and using logarithmic regression analysis, the order of convergence is determined. It is shown that in the proposed method the order of convergence is four, while the family of Newmark methods is second order.

\section{Development of the governing equations and the integration al- gorithm}

The equation of motion for a linear SDOF system is

$$
M \ddot{u}+C \dot{u}+K u=P,
$$

where $M, K$ and $C$ are mass, stiffness and damping, and $P$ is the applied force. The initial conditions are

$$
u(0)=u_{0}, \quad \dot{u}(0)=\dot{u}_{0},
$$

where $u_{0}$ and $\dot{u}_{0}$ are the initial displacement and velocity.

For the $n$th time step, a change of the variable is applied as follows: $\tau=t-t_{n}$ so that, $t \in\left[t_{n}, t_{n+1}\right]$ and, therefore, $\tau \in[0, \Delta t]$, where $\Delta t$ is the time step.

The displacement function is assumed to vary as a complete fourth order polynomial within each time step $\Delta t$ as follows:

$$
u=a_{n} \tau^{4}+b_{n} \tau^{3}+c_{n} \tau^{2}+d_{n} \tau+e_{n},
$$

where $a_{n}$ to $e_{n}$ are the unknown coefficients that should be determined.

Therefore, the velocity and acceleration are defined as

$$
\begin{gathered}
\dot{u}=4 a_{n} \tau^{3}+3 b_{n} \tau^{2}+2 c_{n} \tau+d_{n}, \\
\ddot{u}=12 a_{n} \tau^{2}+6 b_{n} \tau+2 c_{n} .
\end{gathered}
$$

The five coefficients are calculated based on the following conditions. The two initial values of the displacement and velocity based on the previous time step would form two equations

$$
\begin{aligned}
& u(\tau=0)=u_{n}, \quad \text { which yields to } e_{n}=u_{n}, \\
& \dot{u}(\tau=0)=\dot{u}_{n}, \quad \text { which yields to } d_{n}=\dot{u}_{n} .
\end{aligned}
$$


The equation of motion is satisfied at both ends of the time step. (The approximate solution will exactly satisfy the equation of motion at each end of the time step). This means $\ddot{u}_{n}+C \dot{u}_{n}+K u_{n}=P_{n}$ or

$$
M\left(2 c_{n}\right)+C\left(d_{n}\right)+K\left(e_{n}\right)=P_{n} .
$$

From Eqs. (3.4), (3.5) and (3.6) we have

$$
c_{n}=\left(P_{n}-C \dot{u}_{n}-K u_{n}\right) /(2 M),
$$

also $M \ddot{u}_{(n+1)}+C \dot{u}_{(n+1)}+K u_{(n+1)}=P_{(n+1)}$ or

$$
\begin{gathered}
M\left(12 a_{n}(\Delta t)^{2}+6 b_{n}(\Delta t)+2 c_{n}\right)+C\left(4 a_{n}(\Delta t)^{3}+3 b_{n}(\Delta t)^{2}+2 c_{n}(\Delta t)^{+} d_{n}\right)+ \\
K\left(a_{n}(\Delta t)^{4}+b_{n}(\Delta t)^{3}+c_{n}(\Delta t)^{2}+d_{n}\right)=P_{(n+1)} .
\end{gathered}
$$

The last condition requires that the approximate solution has a zero average of the residue upon solving the equation of motion within the time step. It should be noted that this does not necessarily mean that the displacement, velocity or acceleration have a zero average of the error $\int_{0}^{\Delta t}(\operatorname{Re}$ sidue $) d t=0$ or

$$
\int_{0}^{\Delta t}(M \ddot{u}+C \dot{u}+K u-P) d t=0 .
$$

Using Eqs. (3.1)-(3.3), (3.8), and (3.9), the values of $a_{n}, b_{n}$ can be determined as

$$
\left[\begin{array}{ll}
q_{11} & q_{12} \\
q_{21} & q_{22}
\end{array}\right]\left\{\begin{array}{l}
a_{n} \\
b_{b}
\end{array}\right\}=\left\{\begin{array}{l}
r_{n} \\
s_{n}
\end{array}\right\},
$$

where

$$
\begin{gathered}
r_{n}=P_{(n+1)}-c_{n}\left(2 M+2 C(\Delta t)+K(\Delta t)^{2}\right)-d_{n}(C+K(\Delta t))-K e_{n} \\
s_{n}=I_{n}-c_{n}\left(2 M(\Delta t)+C(\Delta t)^{2}+\frac{K}{3}(\Delta t)^{3}\right)-d_{n}\left(C(\Delta t)+\frac{K}{2}(\Delta t)^{2}\right)-e_{n}(K(\Delta t)) \\
I_{n}=\int_{0}^{\Delta t} P\left(\tau+t_{n}\right) d \tau
\end{gathered}
$$

Therefore, $I_{n}$ is the impact due to the applied load in the $n$th time step, and if the load $P$ is known analytically, this integral can be evaluated, however, for complicated loads, the variation of $P$ is assumed to be linear within the time step

$$
P\left(\tau+t_{n}\right)=P_{n}+\left(P_{(n+1)}-P_{n}\right) \tau / \Delta t
$$

Then, Eq. (3.11) becomes

$$
I_{n}=\left(P_{n}+P_{(n+1)}\right) \Delta t / 2,
$$

where the values of $c_{n}, d_{n}, e_{n}$ are known from (3.4)-(3.6), respectively, and the other coefficients in (3.10) are

$$
q_{11}=12 M(\Delta t)^{2}+4 C(\Delta t)^{3}+K(\Delta t)^{4}, \quad q_{12}=6 M(\Delta t)+3 C(\Delta t)^{2}+K(\Delta t)^{3},
$$




$$
q_{21}=4 M(\Delta t)^{3}+C(\Delta t)^{4}+\frac{K}{5}(\Delta t)^{5}, \quad q_{22}=3 M(\Delta t)^{2}+C(\Delta t)^{3}+\frac{K}{4}(\Delta t)^{4} .
$$

Therefore, the values of the two other coefficients are

$$
\left\{\begin{array}{l}
a_{n} \\
b_{n}
\end{array}\right\}=\frac{1}{\operatorname{Det}}\left[\begin{array}{rr}
q_{22} & -q_{12} \\
-q_{21} & q_{11}
\end{array}\right]\left\{\begin{array}{l}
r_{n} \\
s_{n}
\end{array}\right\}
$$

where Det $=q_{11} q_{22}-q_{12} q_{21}$. By using the calculated values of the five parameters of the assumed polynomial for the variation of the displacement, it is possible to calculate the displacement and velocity at the end of the $n$th time step by Eqs. (3.1) and (3.2) as follows:

$$
\begin{gathered}
u_{(n+1)}=a_{n}(\Delta t)^{4}+b_{n}(\Delta t)^{3}+c_{n}(\Delta t)^{2}+d_{n}(\Delta t)+e_{n}, \\
\dot{u}_{(n+1)}=4 a_{n}(\Delta t)^{3}+3 b_{n}(\Delta t)^{2}+2 c_{n}(\Delta t)+d_{n} .
\end{gathered}
$$

Therefore, in each time step the five unknown coefficients are calculated by Eqs. (3.4), (3.5), (3.7), and (3.12) and the values of the displacements and velocity at the end of the time step are calculated by Eqs. (3.13) and (3.14).

\section{Numerical stability}

To check the stability of the method, the magnification matrix is derived and the undamped free vibration of the SDOF system is considered for calculating the eigenvalues of the aforementioned matrix.

Similarly to the other one-step time integration techniques, it is possible to write the values of the displacement and velocity at the end of each time step in terms of those at the beginning of each time step

$$
\left\{\begin{array}{l}
u_{(n+1)} \\
\dot{u}_{(n+1)}
\end{array}\right\}=\left[\begin{array}{ll}
A_{11} & A_{12} \\
A_{21} & A_{22}
\end{array}\right]\left\{\begin{array}{l}
u_{n} \\
\dot{u}_{n}
\end{array}\right\}+\left\{\begin{array}{l}
F_{1} \\
F_{2}
\end{array}\right\}
$$

where the first and the third matrices on the right side of Eq. (4.1) are the magnification and force matrices, respectively. Hence, the magnification matrix is defined by

$$
\left[\begin{array}{ll}
A_{11} & A_{12} \\
A_{21} & A_{22}
\end{array}\right]=\left[\begin{array}{rr}
(\Delta t)^{4} & (\Delta t)^{3} \\
4(\Delta t)^{3} & 3(\Delta t)^{2}
\end{array}\right]\left[\begin{array}{ll}
a_{n 1} & a_{n 2} \\
b_{n 1} & b_{n 2}
\end{array}\right]+\left[\begin{array}{cc}
-\frac{K}{2 M}(\Delta t)^{2}+1 & -\frac{C}{2 M}(\Delta t)^{2}+(\Delta t) \\
-\frac{K}{M}(\Delta t) & -\frac{C}{M}(\Delta t)+1
\end{array}\right]
$$

where

$$
\left[\begin{array}{ll}
a_{n 1} & a_{n 2} \\
b_{n 1} & b_{n 2}
\end{array}\right]=\frac{1}{\operatorname{Det}}\left[\begin{array}{rr}
q_{22} & -q_{12} \\
-q_{21} & q_{11}
\end{array}\right]\left[\begin{array}{ll}
r_{n 1} & r_{n 2} \\
s_{n 1} & s_{n 2}
\end{array}\right]
$$

and

$$
\begin{array}{cc}
r_{n 1}=\frac{K}{2 M}\left(2 C(\Delta t)^{+} K(\Delta t)^{2}\right), & r_{n 2}=\frac{C}{2 M}\left(2 C(\Delta t)^{+} K(\Delta t)^{2}\right)-K(\Delta t), \\
s_{n 1}=\frac{K}{2 M}\left(C(\Delta t)^{2}+\frac{K}{3}(\Delta t)^{3}\right), & s_{n 2}=\frac{C}{2 M}\left(C(\Delta t)^{2}+\frac{K}{3}(\Delta t)^{3}\right)-\frac{K}{2}(\Delta t)^{2} .
\end{array}
$$


The force vector in Eq. (4.1) is defined as

$$
\left\{\begin{array}{l}
F_{1} \\
F_{2}
\end{array}\right\}=\left[\begin{array}{rr}
(\Delta t)^{4} & (\Delta t)^{3} \\
4(\Delta t)^{3} & 3(\Delta t)^{2}
\end{array}\right]\left\{\begin{array}{l}
a_{n f} \\
b_{n f}
\end{array}\right\}+\frac{P_{n}}{2 M}\left\{\begin{array}{c}
(\Delta t)^{2} \\
(\Delta t)
\end{array}\right\}
$$

where

$$
\left\{\begin{array}{l}
a_{n f} \\
b_{n f}
\end{array}\right\}=\frac{1}{\operatorname{Det}}\left[\begin{array}{rr}
q_{22} & -q_{12} \\
-q_{21} & q_{11}
\end{array}\right]\left\{\begin{array}{c}
P_{(n+1)} \\
\Pi
\end{array}\right\} .
$$

For free vibration, the load vector is zero and Eq. (4.1) becomes

$$
\left\{\begin{array}{l}
u_{(n+1)} \\
\dot{u}_{(n+1)}
\end{array}\right\}=[A]\left\{\begin{array}{l}
u_{n} \\
\dot{u}_{n}
\end{array}\right\} \text {. }
$$

The eigenvalues and eigenvectors of $A$ are calculated using $([A]-\lambda[I])\{\Phi\}=\{0\}$ or $\mid A-$ $\lambda I \mid=0$. It is now possible to write $A$ in terms of its eigenvalues and eigenvectors $[A]=$ $[\Phi][\lambda][\Phi]^{-1}$. Here, $[\Phi]$ consists of the eigenvectors of $A$, and $[\lambda]$ is a diagonal matrix consisting the eigenvalues of $A$. Therefore

$$
\left\{\begin{array}{l}
u_{(n+1)} \\
\dot{u}_{(n+1)}
\end{array}\right\}=[\Phi][\lambda]^{(n+1)}[\Phi]^{-1}\left\{\begin{array}{c}
u_{0} \\
\dot{u}_{0}
\end{array}\right\} .
$$

Now to have a stable solution, the norm of the elements of $[\lambda]$ should not be more than unity

$$
\rho(A)=\max \left(\left\|\lambda_{1}\right\|,\left\|\lambda_{2}\right\|\right) \leqslant 1.0 .
$$

In this equation, $\rho(A)$ is the spectral radius, which is a function of the time step length $(\Delta t)$, and also slightly changes with the damping ratio.

To compare the stability of the proposed method to the members of the Newmark method, the amplification matrices for those methods derived in [18] are used.

Figure 4.1 presents the comparison of the spectral radius of the proposed method to those of the other methods, which shows that the proposed method is stable for time steps of up to $1.24 T$.

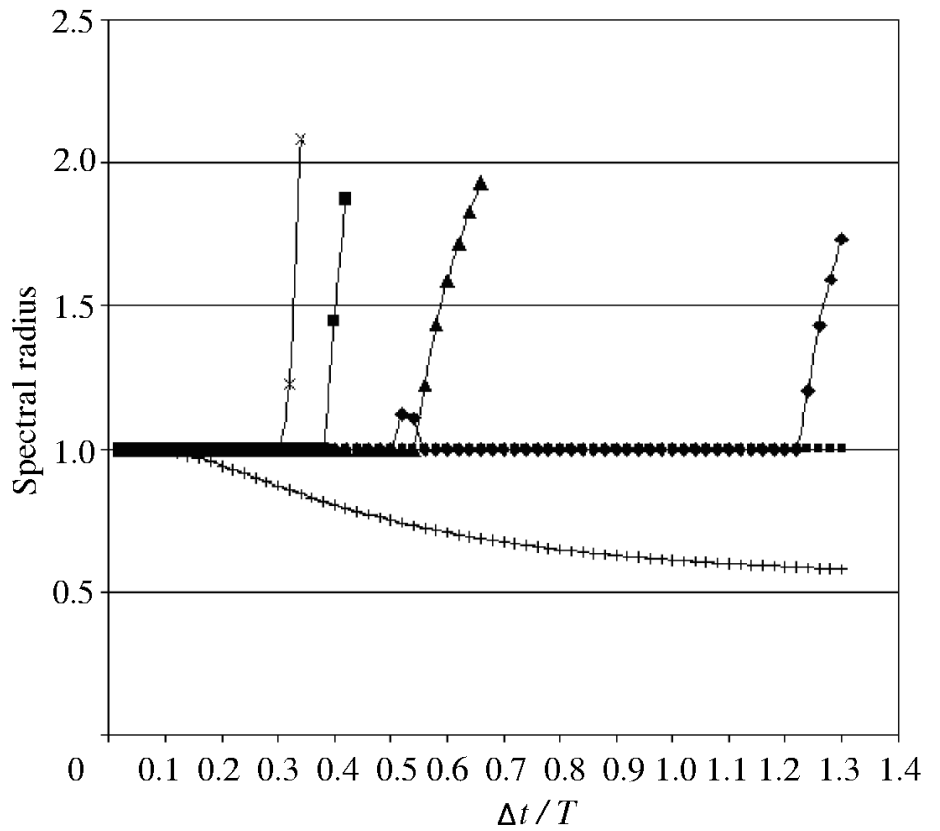

Fig. 4.1. Comparison of the spectral radius between the proposed and conventional methods $(\xi=0):-$ proposed method; average acceleration method; $\boldsymbol{\Lambda}$ - linear acceleration method; $\times$ - central difference method; - Fox - Goodwin method; + Whilson-theta method 
Figure 4.1 also shows that all nondissipative conditionally stable methods (the proposed method, the linear acceleration, the central finite difference and the Fox-Goodwin) method have a spectral radius of unity up to their critical time steps after which it increases without bound. On the other hand, for dissipative unconditionally stable methods like the Wilson$\theta$, spectral radius is always less than unity and for non-dissipative unconditionally stable methods, like the average acceleration method it is always equal to one. It should also be noted that the CTS for the proposed method is much larger than those for the other methods.

Finally, it is important to note that the proposed method suffers a local instability for the range of $\Delta t / T$ between 0.51 and 0.55 , and after that stability is gained. A similar situation is observed in the method presented in [37]. This local instability is only true for undamped behavior, and for $\xi \geqslant 5 \%$ this local instability does not exist, as is shown in Fig. 4.2.

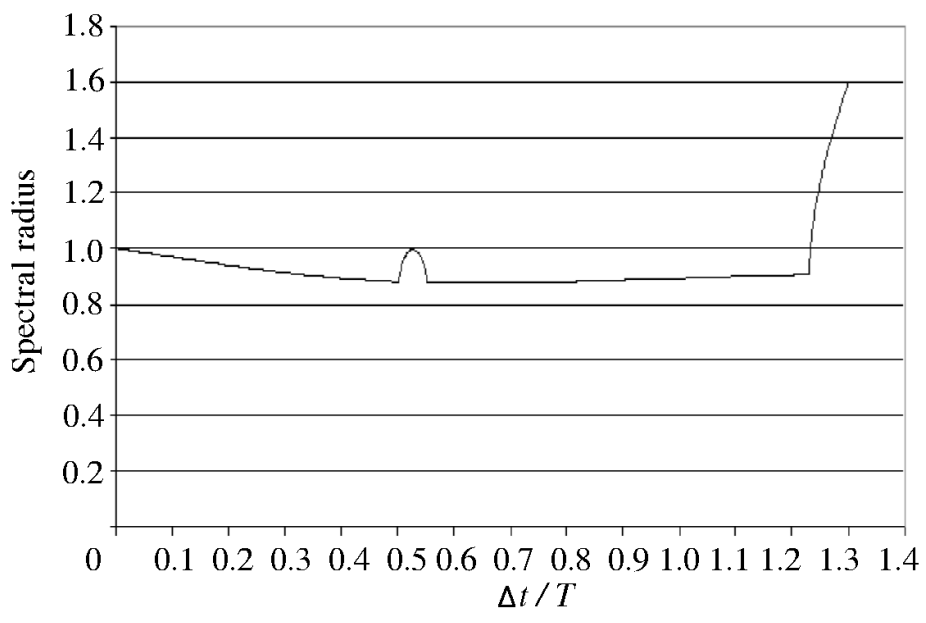

Fig. 4.2. Spectral radius of the proposed method $(\xi=5 \%)$

\section{Numerical accuracy}

The accuracy evaluation of the time integration methods requires assessments of two types of error (dissipation and dispersion), which are calculated as functions of $\Delta t / T$. These errors are determined in terms of the eigenvalues of the amplification matrix as follows:

$$
\lambda_{1,2}=e^{-\bar{\omega} \Delta t(\bar{\xi} \pm i)}
$$

where $\lambda_{1,2}$ denotes the complex conjugate eigenvalues of the amplification matrix and $\bar{\omega}, \bar{\xi}$ stand for the numerical frequency and numerical damping induced by the time integration method. Equation 45 can be rewritten in the form

$$
\bar{\omega}=\frac{1}{\Delta t} \operatorname{tg}^{-1}\left(\frac{\operatorname{Im} g\left(\lambda_{1,2}\right)}{\operatorname{Re} a l\left(\lambda_{1,2}\right)}\right)
$$

and

$$
\bar{\xi}=-\frac{1}{\bar{\omega} \Delta t} \operatorname{Ln}\left\|\lambda_{1,2}\right\|
$$

Im $g$ and Re $a l$ in (5.1) represent the imaginary and the real parts of the eigenvalues, respectively. Equation (5.2) is employed to examine the accuracy of the proposed method by evaluating it using the norm of the eigenvalues $\left\|\lambda_{1,2}\right\|$ as follows: 1) for $\left\|\lambda_{1,2}\right\|$ less than unity, 
the equation yields to positive numerical damping; 2) for $\left\|\lambda_{1,2}\right\|$ greater than unity, the numerical damping is negative, which makes the solution become unstable; and for $\left\|\lambda_{1,2}\right\|$ equal to one, there will be no numerical damping.

To calculate the period error (dispersion), we can write

$$
\bar{T}=2 \pi / \operatorname{bar} \omega .
$$

Here $\bar{T}$ is the period of the numerical solution and, therefore, the period error would be equal to

$$
P E=\frac{\bar{T}-T}{T}=\frac{\bar{T}}{T}-1
$$

Equation (4.2) shows that the size of the amplification matrix of the proposed method is $2 \times 2$ and the complex eigenvalues are conjugate. The size of this matrix for the Newmark family of methods, on the other hand, is $3 \times 3$, and it always has a real eigenvalue (called a spurious root and it is not considered) in addition to the pair of complex conjugate roots, which are taken into account for stability and accuracy evaluations.

Figure 5.1 compares the numerical damping of the proposed method to those of the other methods listed in the graph. It is seen that all Newmark methods, for which $\gamma=0.5$, are nondissipative up to their CTS, and then they have positive numerical damping (except for the average acceleration that is always nondissipative). The proposed method follows the same behavior with larger CTS.

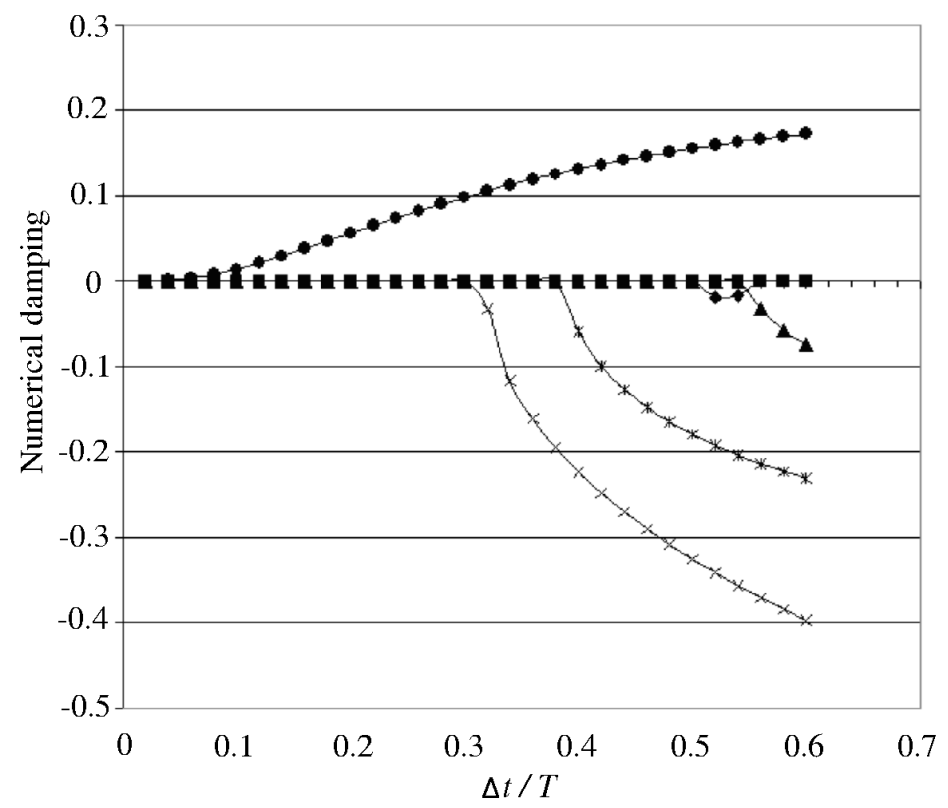

Fig. 5.1. Numerical damping (dissipation) of the proposed method compared to other methods: - proposed method; - - average acceleration method; $\boldsymbol{\Delta}$ - linear acceleration method; $\times$ - central difference method; $*$ - Fox — Goodwin method; • - Whilson-theta method

Figure 5.2 compares the amplitude error of the proposed method and other methods. Here it is seen that the dispersion of the proposed method is much less than that of the others and will elongate the period like the average acceleration method and the Wilson- $\theta$ method, but in the central difference and Fox - Goodwin methods, period is shortened. 
Fig. 5.2. Period error (dispersion) of the proposed method compared to other methods: - proposed method;

age acceleration method; $\boldsymbol{\Delta}$ - linear acceleration method; $\times$ - central difference method; * - Fox — Goodwin method; + - Whilson-theta method

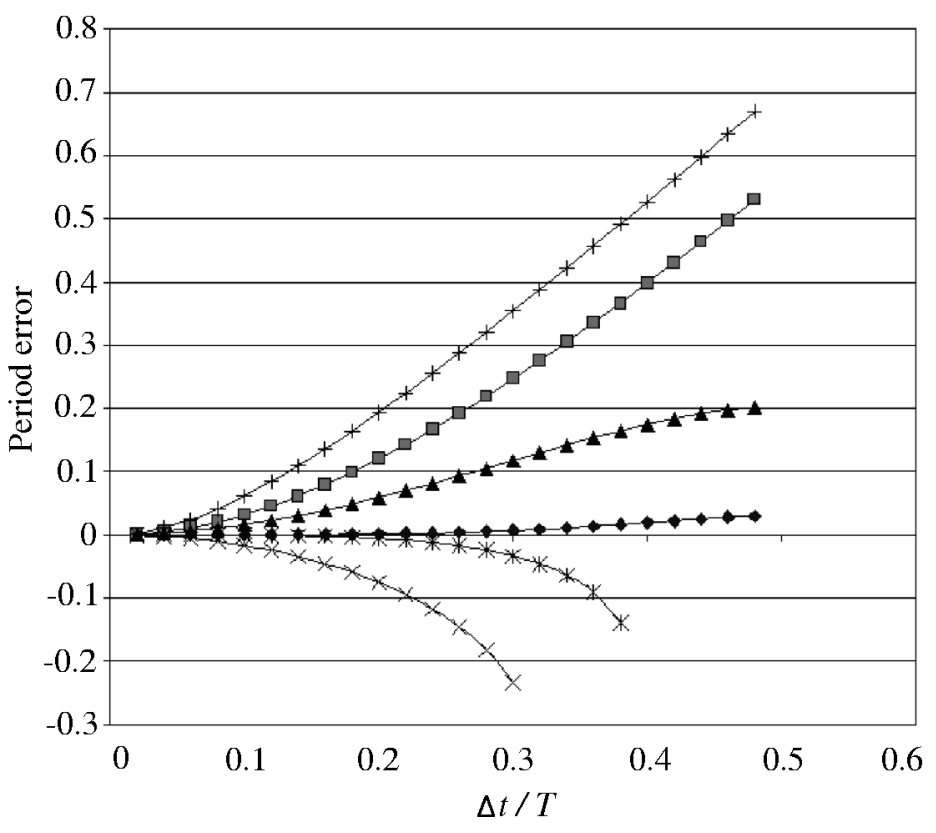

Figure 5.3 shows the accuracy of the proposed method for harmonic excitations. The error of the magnification factor for the displacement calculated using the proposed method and the other methods listed in the graph are compared to show that the error of the proposed method is much smaller than in the other methods. Here $W_{0}, W$ are the frequency of the applied load and the natural frequency of the SDOF system.

Fig. 5.3. Error of the spectral displacement magnification factor for harmonic load $(\xi=$ $0.05, \Delta t / T=0.1): \diamond$ - proposed method; - average acceleration method;

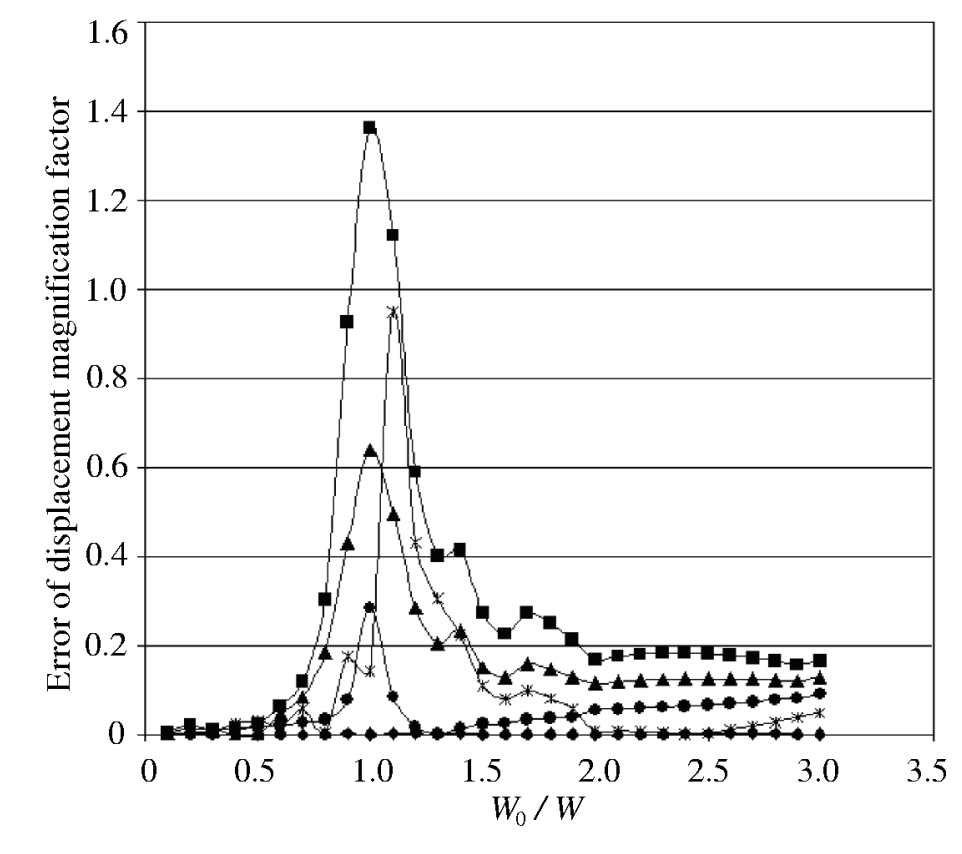
linear acceleration method; $\times$ - central difference method; • - Fox — Goodwin method

\section{Numerical convergence}

To evaluate the order of convergence, a constant time is considered and the error for a different number of time steps (different sizes of time steps), displacement error, is calculated and using the regression analysis, the best value of the power is determined:

$$
\left|\left(u_{n}-u_{n}^{e x}\right)\right|=c(\Delta t / T)^{\alpha} .
$$


Here $u_{n}$ and $u_{n}^{e x}$ are the approximate and exact values of the displacement at the specified time, $t_{n}$ and $c, \alpha$ are constants to be determined using the regression analysis for different values of time step sizes. $\alpha$ is called the order of convergence. In Fig. 6.1 the values of the order of the convergence for different values of frequency ratios are plotted and it is seen that the proposed method has an order of convergence of more than four, while the family of Newmark methods are about second order. (Fox - Goodwin method has the third order of convergence, it is an exception and maybe for this reason is called Royal Road method).

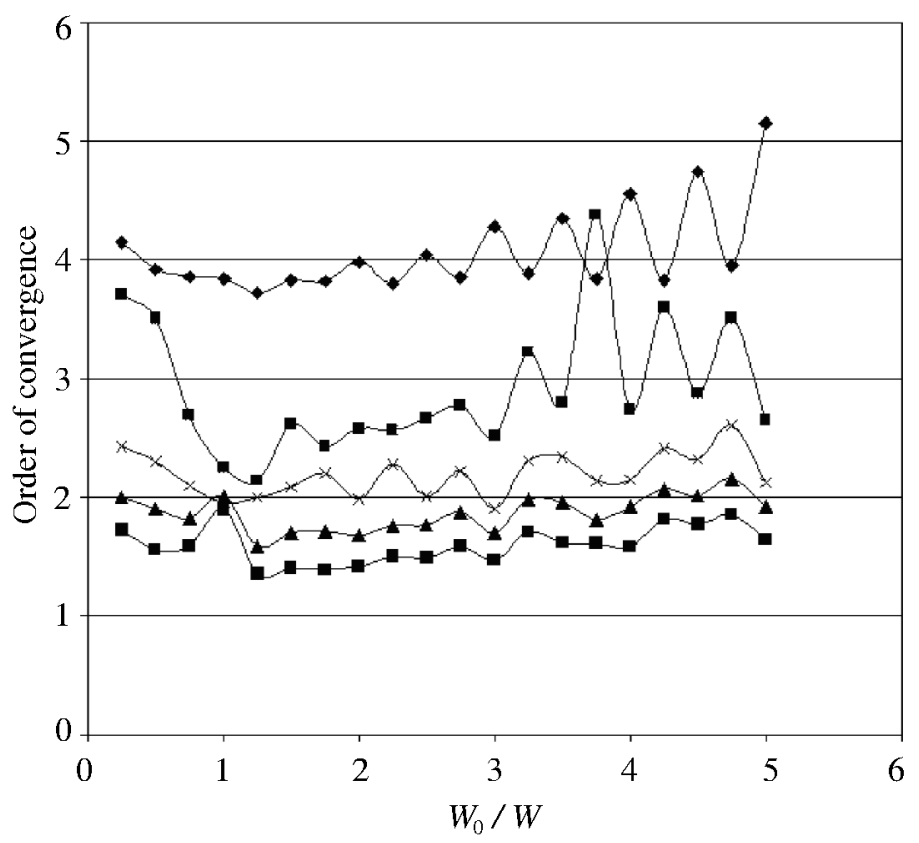

Fig. 6.1. Order of convergence for the proposed method compared to other methods: $\checkmark$ - proposed method; $\mathbf{\square}$ - average acceleration method; $\boldsymbol{\Delta}$ - linear acceleration method; $\times$ - central difference method;

Fox - Goodwin method

The fourth order convergence means that upon halving the time step size the displacement error will be sixteen times smaller.

\section{Conclusions}

A new method of time integration for structural dynamics is proposed in which the displacement in each time step is assumed to be a fourth order polynomial (therefore, the acceleration is quadratic in time). The polynomial has five unknown coefficients and to calculate them five equations are required. The equations are as follows: two initial conditions from the previous time step, two equations from the satisfying of the equation of motion at both ends of the time step, and one equation based on the equating of the weighted average of the residual (with the weight function of unity), within the time step, to zero. The proposed method has higher stability, accuracy, and the rate of convergence compared to other methods. The critical time step ( the time step beyond which numerical instability occurs) for the proposed method is 1.24 times the natural period of the structure, while the critical time step for linear acceleration, central difference, and Fox - Goodwin methods are 0.55, 0.32 and 0.39 , respectively. Also this method is non-dissipative and its dispersion error is significantly less than in other methods. The order of convergence for the proposed method is 4 compared to 2 for the Newmark methods. 


\section{Appendix}

To verify the results of the method, exact solutions are required. For a harmonic load, a close form solution is available but, for the general case of loading, the Duhamel integral is considered;

$$
u\left(t_{(n+1)}\right)=\frac{1}{M \omega_{D}} \int_{0}^{t_{(n+1)}} P(\tau) e^{-\zeta \omega_{n}\left(t_{(n+1)}-\tau\right)} \sin \left[\omega_{D}\left(t_{(n+)}-\tau\right)\right] d \tau
$$

and if the load is assumed to vary linearly in each time step $P_{i}(\tau)=a_{i} \tau+b_{i}$. Equation (8.1) can be written as

$$
u\left(t_{(n+1)}\right)=\frac{1}{M \omega_{D}} \sum_{i=0}^{n} \int_{t_{i}}^{t_{(i+1)}}\left(a_{i} \tau+b_{i}\right) e^{-\zeta \omega_{D}\left(t_{(n+1)}-\tau\right)} \sin \left[\omega_{D}\left(t_{(n+1)}-\tau\right)\right] d \tau .
$$

On the other hand, we have the close form solutions of these two integrals

$$
\int X e^{(a X)} \sin (b X) d X=\frac{e^{(a X)}}{a^{2}+b^{2}}\left[\left(a X-\frac{a^{2}-b^{2}}{a^{2}+b^{2}}\right) \sin (b X)-\left(b X-\frac{2 a b}{a^{2}+b^{2}}\right) \cos (b X)\right]
$$

and

$$
\int e^{(a X)} \sin (b X) d X=\frac{e^{(a X)}(a \sin (b X)-b \cos (b X))}{a^{2}+b^{2}} .
$$

Hence, the exact solution is determined in closed form. This means that for the general case of loading, it is also possible to have the exact solution.

\section{References}

1. J. D. Achenbach, Wave propagation in elastic solids. North-Holland, 1999.

2. A. Kukreti and H. Issa, Dynamic analysis of nonlinear structures by pseudo-normal mode superposition method, Computers \& Structures, 19 (1984), no 4, pp. 653-663.

3. J. D. Houbolt, A recurrence matrix solution for the dynamic response of elastic aircraft, Journal of Aerospace Science, 17 (1950), pp. 540-550.

4. T. Belytschko and Y. Lu, Explicit multi-time step integration for first and second order finite element semidiscretizations, Computer Methods in Applied Mechanics and Engineering, 108 (1993), no 3-4, pp. 353383.

5. P. Smolinski, S. Sleith, and T. Belytschko, Stability of an explicit multi-time step integration algorithm for linear structural dynamics equations, Computational Mechanics, 18 (1996), no 3, pp. 236-244.

6. P. Smolinski and Y.S. Wu, An implicit multi-time step integration method for structural dynamics problems, Computational Mechanics, 22 (1998), pp. 337-343.

7. M. A. Dokainish and K.Subbaraj, A survey of direct time integration methods in computational structural dynamics. I, Explicit methods. Computers \& Structures, 32 (1989), no 6, pp. 1371-1386.

8. G.D.Hahn, A modified Euler method for dynamic analysis, International Journal for Numerical Methods in Engineering, 32 (1991), no 5, pp. 943-955.

9. T. J. R. Hughes and T. Belytschko, A précis of developments in computational methods for transient analysis, Journal of Applied Mechanics, 50 (1983), pp. 1033-1041.

10. K. C. Park, Practical aspects of numerical time integratio, Computers \& Structures, 7 (1977), pp. 343353.

11. K. Subbaraj and M.A. Dokainish, A survey of direct time integration methods in computational structural dynamics. II. Implicit methods, Computers \& Structures, 32 (1989), no 6, pp. 1387-1401.

12. W. L. Wood, A further look at Newmark, Houbolt, etc., time-stepping formulae, International Journal for Numerical Methods in Engineering, 20 (1984), pp. 1009-1017.

13. T. Belytschko and R. Mullen, Stability of explicit-implicit mesh partitions in time integration, International Journal for Numerical Methods in Engineering, pp. 1575-1586. 
14. T. J. R. Hughes and W. K. Liu,Implicit-explicit finite elements in transient analysis: Stability theory, Journal of Applied Mechanics, 45 (1978), pp. 371-374.

15. J. Chung and G. M. Hulbert, A time integration algorithm for structural dynamics with improved numerical dissipation: The generalized- $\alpha$ method, Journal of Applied Mechanics, 60 (1993), pp. 371-375.

16. H. Hilber, T.J.R. Hughes, and R. L. Taylor, Improved numerical dissipation for time integration algorithms in structural dynamics, Earthquake Engineering and Structural Dynamics, (1977), no 5, pp. 283292 .

17. H. Hilber and T. J. R. Hughes, Collocation, dissipation and "overshoot" for time integration schemes in structural dynamics, Earthquake Engineering and Structural Dynamics, 6 (1978), pp. 99-117.

18. K. J. Bathe and E. L. Wilson, Stability and accuracy analysis of direct time integration methods, Earthquake Engineering and Structural Dynamics, 1 (1973), pp. 283-291.

19. T. Belytschko and T. Hughes, Computational methods in mechanics. Vol. 1. Computational Methods for Transient Analysis. North-Holland. 2001.

20. J. V. Neumann and R. D. Richtmyer, A method for the numerical calculation of hydrodynamic shocks, Journal of Applied Physics, 21 (1950), pp. 232-257.

21. K. C. Park, An improved stiffly stable method for direct integration of nonlinear structural dynamic equations, Journal of Applied Mechanics Division, (1975), pp. 464-470.

22. N. M. Newmark, A method of computation for structural dynamics, Journal of the Engineering Mechanics Division, 85 (1959), no 3, pp. 67-94.

23. M. Austin, High-order integration of smooth dynamical systems: Theory and numerical experiments, International Journal for Numerical Methods in Engineering, 36 (1993), pp. 2107-2122.

24. S. Pezeshk and C. V. Camp, An explicit time integration technique for dynamic analysis, International Journal for Numerical Methods in Engineering, 38 (1995), pp. 2268-2281.

25. G. Sheng and B. Liu, Modified Newmark method for head-disk interface dynamics, Computers \& Structures, 66 (1998), no 2-3, pp. 145-154.

26. M. Katona, A unified set of single step algorithms. Part 1: General formulation and applications, International Journal for Numerical Methods in Engineering, 20 (1984), pp. 1529-1552.

27. M. A. Katona and O.C.Zienkiewicz, A unified set of single step algorithms. Part 3: The beta-m method, a generalization of the Newmark scheme, International Journal for Numerical Methods in Engineering, 21 (1985), pp. 1345-1359.

28. O. C. Zienkiewicz and R. W. Lewis, An analysis of various time-stepping schemes for initial value problems, Earthquake Engineering and Structural Dynamics, 1 (1973), pp. 407-408.

29. O. C. Zienkiewicz, W. L. Wood,N. W. Hine, and R. L. Taylor, A unified set of single step algorithms. Part 1: General formulation and applications, International Journal for Numerical Methods in Engineering, 20 (1984), pp. 1529-1552.

30. S. Chen, J. Hansen, and D. Tortorelli, Unconditionally energy stable implicit time integration: application to multibody system analysis and design, International Journal for Numerical Methods in Engineering, 48 (2000), pp. 791-822.

31. T.C.Fung, Weighting parameters for time-step integration algorithms with predetermined coefficients, International Journal for Numerical Methods in Engineering, 49 (2000), pp. 1397-1430.

32. E. Kirchner and B.Simeon, A higher-order time integration method for viscoplasticity, Computer Methods in Applied Mechanics and Engineering, 175 (1999), pp. 1-18.

33. P. Smolinski, Subcycling integration with non-integer time steps for structural dynamics problems, Computers \& Structures, 59 (1996), no 2, pp. 273-281.

34. L. Zhang, J. W.Zu, and Z.Zheng, The stochastic Newmark algorithm for random analysis of multidegree-of-freedom nonlinear systems, Computers \& Structures, 70 (1999), pp. 557-568.

35. B. W. Golley, A weighted residual development of a time-stepping algorithm for structural dynamics using two general weight functions, International Journal for Numerical Methods in Engineering, 42 (1998), pp. 93-103.

36. K. Tamma and J.D'costa, A new explicit variable time-integration self-starting methodology for computational structural dynamics, International Journal for Numerical Methods in Engineering, 33 (1992), pp. 1165-1180.

37. B. W. Golley, A time-stepping procedure for structural dynamics using Gauss point collocation, International Journal for Numerical Methods in Engineering, 39 (1996), pp. 3985-3998. 